\title{
Cardiac resynchronisation in congenital heart disease
}

\author{
Jan Janoušek \\ Kardiocentrum and Cardiovascular Research Center, Department of Cardiology, University Hospital Motol, Prague, Czech Republic
}

Address: prof. Jan Janoušek, M.D., Ph.D., Dětské kardiocentrum, University Hospital Motol, V Úvalu 84, 15006 Prague 5, Czech Republic, e-mail: jan.janousek@lfmotol.cuni.cz

Cardiac resynchronization therapy (CRT) is a routine treatment option for adult patients with chronic left ventricular (LV) failure due to idiopathic or ischaemic dilated cardiomyopathy associated with electromechanical dyssynchrony. Initial studies showed acute hemodynamic efficacy and subsequent controlled randomised trials confirmed reverse LV remodelling, functional improvement, decrease in heart failure hospitalisation, and decreased overall mortality. In both European ${ }^{1}$ and North American ${ }^{2}$ guidelines CRT is a class I (level of evidence A) therapy for patients with an LV ejection fraction $\leq 35 \%$ and QRS $\geq 120 \mathrm{~ms}$ who remain symptomatic (New York Heart Association [NYHA] functional class III-IV) despite optimal medical treatment. Smaller retrospective series, including two multi-centre surveys, ${ }^{3,4}$ have meanwhile shown a benefit of CRT in patients with congenital heart disease both for the acute manipulation of cardiac output after surgery for congenital heart defects, and for the management of chronic systemic ventricular failure. This review will summarise the use of temporary and permanent CRT on the background of the heterogeneity of this population in terms of age, structure and causes of electromechanical dyssynchrony, and give some practical hints for CRT application.

\section{Pathophysiology of electromechanical dyssynchrony}

Electromechanical dyssynchrony causes a sequence of events which may result in pathological ventricular remodelling and eventually failure. These have been documented in animal experiments and confirmed subsequently in the clinical setting. Wymen et $\mathrm{al}^{5}$ studied mechanical LV deformation by segmental circumferential strain in dogs paced from the right ventricular (RV) apex. They found early contraction close to the pacing site with accompanying stretch of the remote segments, followed by subsequent contraction of late activated segments along with relaxation and stretch of the early activated sites. The group of Prinzen et al was able to show the consequences of dyssynchronous activation on local myocardial work with decrease in the early sites contracting with a low local preload and increase in the late sites whose preload was increased by preceding stretch. ${ }^{6}$ Finally, van Oosterhout et al ${ }^{7}$ showed development of asymmetric myocardial hypertrophy due to differences in local myocardial work with decrease in regional wall thickness and volume at the early contracting sites and increase in the areas of late contraction.

Clinical observations in children have confirmed the experimentally described contraction patterns ${ }^{8}$ (Figure 1) and provided empiric proof of wasted myocardial work caused by ventricular dyssynchrony (Figure 2). Published data have also shown that restoration of a normal mechanical contraction pattern will increase contraction efficiency. ${ }^{9}$ Response patterns to CRT should obviously be dependent on the aetiology of ventricular failure. Fast and almost complete reverse ventricular remodelling was observed in patients with primary LV dyssynchrony and failure caused solely by RV pacing. ${ }^{10}$ Less optimal results, however, may be obtained in those patients in whom dyssynchronous contraction is a consequence or companion of other heart disease. Thus, the efficacy of CRT will vary with the underlying structural and pathophysiologic substrate, such as the anatomy of the systemic ventricle (left, right or single), the presence and degree of structural systemic atrioventricular (AV) valve regurgitation, and the presence of primary myocardial disease or scarring. Potential response patterns to CRT are depicted in Figure 3. 


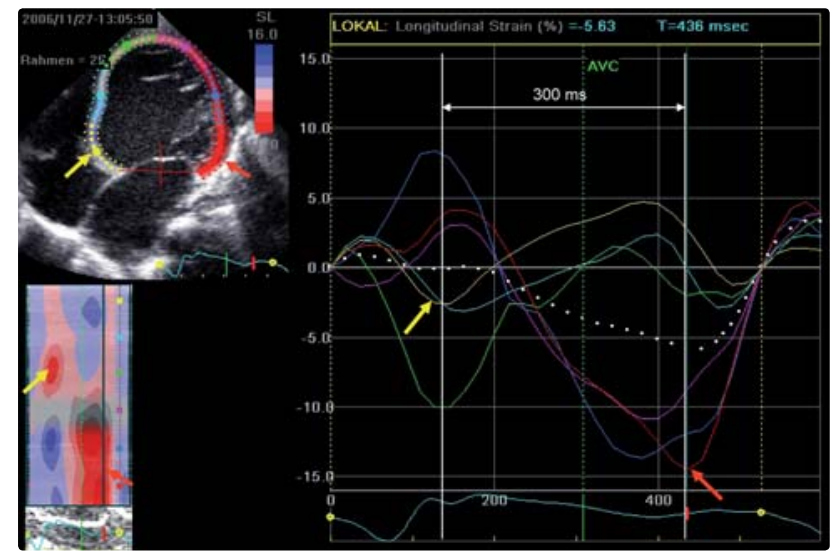

Figure 1 New echocardiographic technique of speckle tracking derived two dimensional strain is able to detect segmental myocardial deformation. The upper left panel displays colour coded left ventricular (LV) segments in the apical four chamber view with corresponding strain curves in the right hand panel (negative strain = contraction; positive strain = relaxation or stretch) and curved two dimensional strain $\mathrm{M}$ mode in the lower left panel. During right ventricular outflow tract pacing for complete atrioventricular (AV) block, early contraction appears in the basal and mid ventricular septum (coded yellow and light blue; yellow arrow) causing stretch of the corresponding segments on the LV free wall (coded red and dark blue) followed by late contraction of the latter at the time of septal relaxation (red arrow). This results in an excessive septal to free wall mechanical delay of $300 \mathrm{~ms}$ and severe LV dyssynchrony.

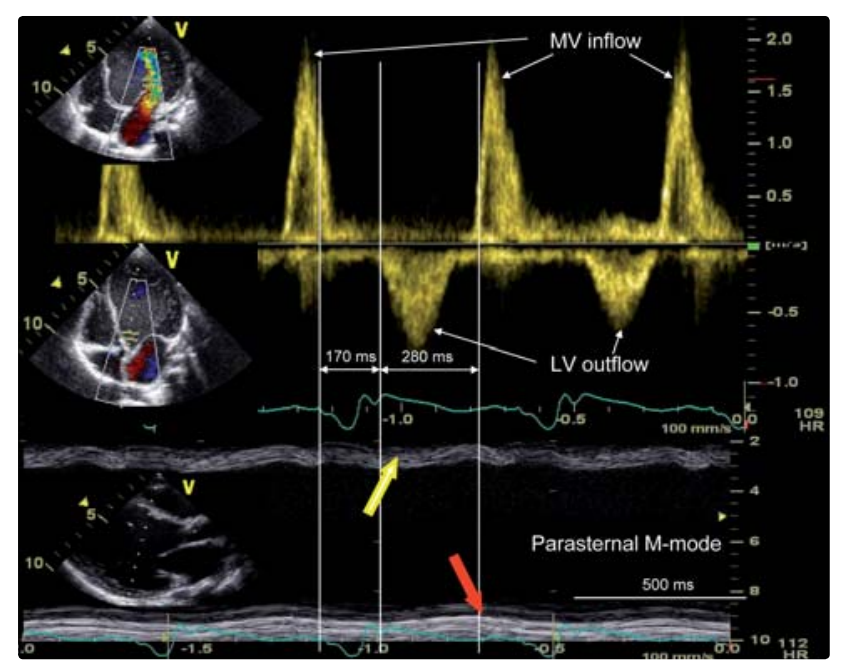

Figure 2 The same patient as in Figure 1. Mounted picture using different echocardiographic techniques aligned to the same time scale to display global left ventricular (LV) timing. From top to bottom: pulsed Doppler from the mitral valve, pulsed Doppler from the LV outflow tract, ECG, M mode from the parasternal long axis view. The LV pre-ejection period of $170 \mathrm{~ms}$ reflects slow systolic LV pressure rise due to dyssynchrony evident by a septal (yellow arrow) to posterior (red arrow) wall motion delay of $280 \mathrm{~ms}$. Ejection (see LV outflow Doppler tracing) starts by septal contraction at the time of maximum end-diastolic stretch of the LV posterior wall. Peak LV posterior wall contraction appears after aortic valve closure (end of ejection as depicted by the LV outlow Doppler) and corresponds with onset of the LV filling (mitral valve inflow). Energy put into LV posterior wall contraction is partially wasted because it is not contributing to LV ejection.

$\mathrm{LV}$ - left ventricle, MV - mitral valve

\section{Epidemiology of electromechanical dyssynchrony with respect to CRT}

Comprehensive data on the prevalence of cardiac dyssynchrony amenable to CRT have not yet been published, but information can be retrieved from various sources. First, looking at the two available multicentre surveys on CRT in children and patients with congenital heart disease $e^{3,4}$ (Table 1), the presence of a left bundle branch block with left ventricular dysfunction (the most frequent indication for CRT in adult heart disease) is rather uncommon (9.2\%) as an indication for CRT in the congenital heart disease population. In one half to two thirds of the cases, CRT was applied as an upgrade of conventional ventricular pacing because of pacing associated heart failure; $70-80 \%$ of the patients had structural congenital heart disease with a systemic RV in up to a third. Additional data are specifically available for the systemic RV population, with $9.3 \%$ of the patients after the atrial baffle procedure for $\mathrm{d}$-transposition

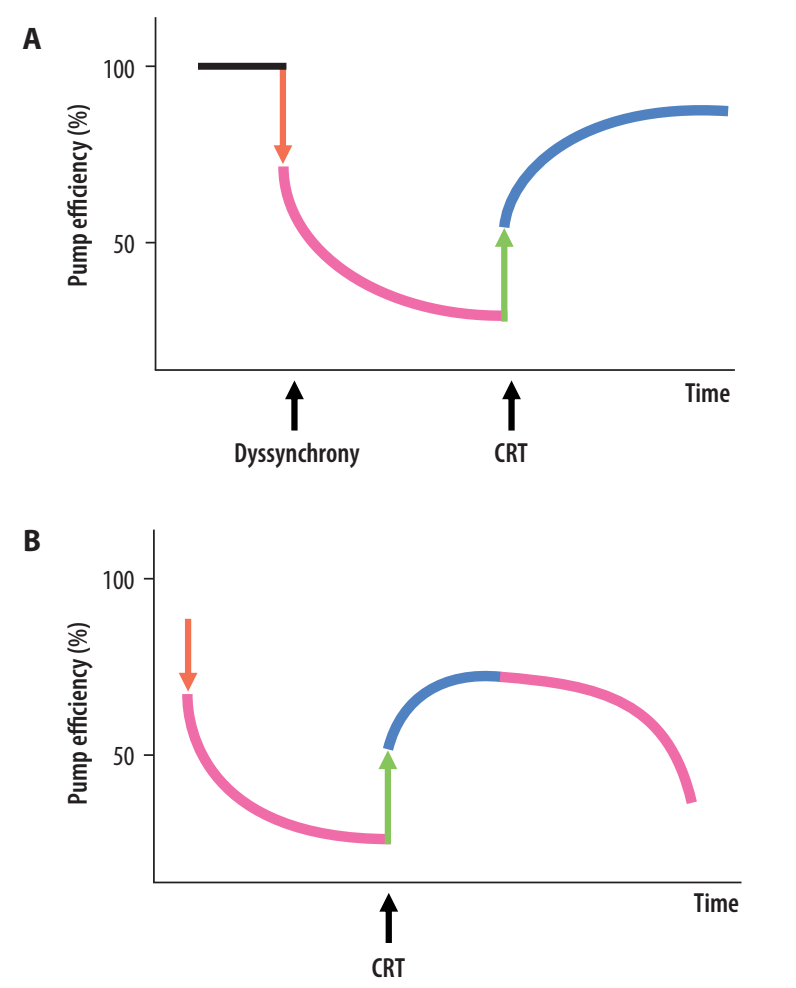

Figure 3 Two potential response patterns to cardiac resynchronisation therapy (CRT). (A) Ventricular failure was caused exclusively by dyssynchrony (for instance, right ventricular [RV] pacing induced dyssynchrony in an otherwise normal heart). Dyssynchrony causes an immediate decrease in pump efficiency (orange arrow) followed by pathologic left ventricular (LV) remodelling (pink) leading to severe ventricular failure. CRT causes immediate improvement (green arrow) followed by almost complete reverse remodelling (blue).

(B) Dyssynchrony is a consequence or companion of other myocardial disease (for instance, bundle branch block in idiopathic dilated cardiomyopathy). Pathologic remodelling progresses (pink) until CRT is started. CRT leads to immediate improvement in ventricular function (green arrow) followed by a variable degree of reverse LV remodelling (blue), but finally the original disease process prevails and results in further worsening of ventricular function (pink). 
Table 1 Demographic data from two multicentre surveys on cardiac resynchronization therapy (CRT) in children and patients with congenital heart disease

\begin{tabular}{|c|c|c|c|c|c|c|c|}
\hline Study & $\mathrm{n}$ & $\begin{array}{l}\text { Median age } \\
\text { (years) }\end{array}$ & SHD (\%) & $\begin{array}{l}\text { Systemic } \\
\text { RV (\%) }\end{array}$ & $\begin{array}{l}\text { Functional } \\
\text { SV (\%) }\end{array}$ & Conv pacing (\%) & $\begin{array}{l}\text { LBBB } \\
\text { systemic LV (\%) }\end{array}$ \\
\hline Dubin et $\mathrm{al}^{3}$ & 103 & 12.8 & 71.0 & 16.5 & 6.8 & 44.7 & - \\
\hline Janoušek et al ${ }^{4}$ & 109 & 16.9 & 79.8 & 33.0 & 3.7 & 77.1 & 9.2 \\
\hline
\end{tabular}

of great arteries and $6.1 \%$ of patients with congenitally corrected transposition fulfilling the currently applied CRT indication criteria if including NYHA class II patients. ${ }^{11}$ Thus, the congenital heart disease cohort amenable to CRT is completely different from its adult idiopathic or ischaemic heart disease counterpart, and data from the large adult CRT trials cannot be easily transferred to this specific population.

\section{Principles of CRT}

Although still not reflected by the currently used indication criteria, CRT is aimed at correction of ventricular mechanical dyssynchrony ${ }^{12}$ and several studies have shown this to be a prerequisite for CRT efficacy. Figure 4 displays a simple scheme of CRT in a patient with left bundle branch block causing a septal to LV free wall electrical and mechanical

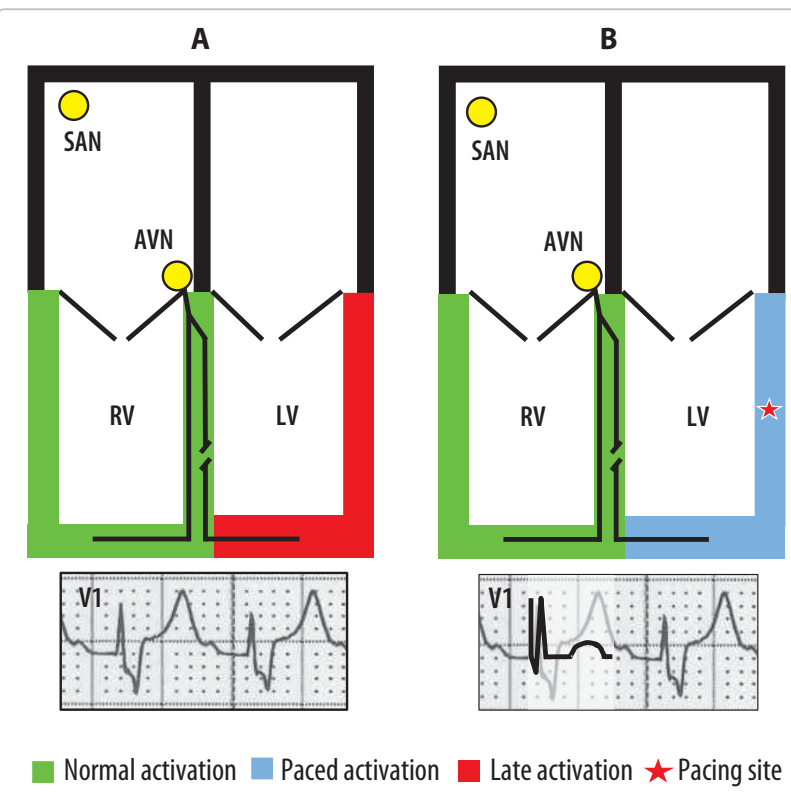

Figure 4 Simple scheme of cardiac resynchronisation therapy (CRT) in a patient with left bundle branch block and left ventricular (LV) dysfunction. (A) During native conduction electrical and mechanical LV free wall activation is delayed because of the left bundle branch block. This results in septal to lateral LV dyssynchrony. (B) CRT is applied by properly timed LV free wall pre-excitation and results in fusion between spontaneous (septal) and paced (free wall) activation reflected by a narrowed QRS complex and restoration of LV contraction synchrony. Single site LV or biventricular pacing may be used to achieve this goal (see text for details).

AVN - atrioventricular node, LV - left ventricle, RV - right ventricle, SAN - sinoatrial node delay. Although anatomical and functional situations may vary, this scheme is generally valid for any CRT application. CRT has mostly been realised by biventricular pacing, but single site atrial synchronised pacing of the late activated myocardial segments in fusion with spontaneous ventricular activation may be as effective. ${ }^{13}$ The reasons for the prevailing use of biventricular pacing lie in the inability to optimize the mechanical AV delay by single site pacing in case of prolonged spontaneous AV conduction (excessive shortening of AV delay at the free wall pacing site would lead to inverse dyssynchrony with interventricular septum being activated to late) and in difficulties with appropriate $\mathrm{AV}$ delay adaptation during exercise (pacing may occur too late or too early to achieve effective fusion with spontaneous activation). In the case of biventricular pacing one of the leads is generally placed in or on the subpulmonary ventricle close to interventricular septum and the other lead is located over the systemic ventricular free wall. There should be a sufficient spatial and electrical separation between both leads so that the resynchronised ventricle lies in between the two electrodes. If pacing from one of the leads, local activation should occur late (at the end of the QRS complex) in the other. Generally, different combinations of transvenous and thoracotomy leads may be used in adaptation to patient's age and cardiac anatomy to achieve this goal

Echocardiographic techniques may help to diagnose and quantify mechanical dyssynchrony and to identify areas of late mechanical activation. Unfortunately, none of them has been so far validated in larger studies and the reproducibility of echocardiograhic techniques has recently been questioned in the PROSPECT trial. ${ }^{14}$ However, careful integration of available data on the electrical and mechanical activation sequence, myocardial viability and global cardiac timing (Figures 1 and 2) may still be helpful in individual cases to support CRT indication and to guide lead placement. Newer echocardiographic modalities like the speckle tracking have already been shown to predict CRT efficacy ${ }^{15}$ and may hold promise for the future.

\section{Temporary CRT for treatment of acute postoperative heart failure}

The use of CRT for managing low cardiac output after surgery for congenital heart disease has been under study for almost a decade. A recent publication ${ }^{10}$ summarised briefly current data illustrating the anatomic and functional heterogeneity of 
the treated populations. To achieve synchrony of ventricular contraction either atrial synchronous multisite ventricular pacing or single site pacing at the site of late activation with fusion with spontaneous depolarisation is used. In the latter case, the AV delay has to be manipulated to achieve the shortest QRS duration possible by placing the pacing pulse at the beginning of the spontaneous QRS complex.

All available studies ${ }^{10}$ reported haemodynamic improvement. Three of them focused partially or totally on the resynchronization of a failing subpulmonary right ventricle (RV). Fusion pacing to one or more RV free wall sites was adjusted to functionally alleviate right bundle branch block. Successful resynchronisation of a single ventricle was reported in two studies. Three ventricular leads were placed as far apart as possible on the apex, right free wall and either outflow tract, or left free wall. One study showed that mechanical resynchronisation of a single ventricle could be achieved despite normal baseline QRS duration (mean $94 \mathrm{~ms}$ ). This has opened a space for further research looking at mechanical dyssynchrony as a potential and correctable substrate contributing to heart failure in single ventricular patients.

In some of the reported patients temporary resynchronisation was a very powerful tool enabling discontinuation from cardiopulmonary bypass when all other measures were unsuccessful. The method has a potential for interrupting the vicious circle of dyssynchrony associated acute heart failure. In this situation, an increase in catecholamine support leads to exaggerated heart rates due to sinus tachycardia, and results in a combination of inefficient dyssynchronous ventricular contraction with severe limitation of diastolic ventricular filling due to short cardiac cycle duration and prolongation of systole and both isovolumic periods. Cooperation with the cardiac surgeon, identification of the failing and resynchronisable ventricle (either systemic or subpulmonary), and appropriate placement of temporary pacing wires at areas of late electromechanical activation is, however, essential. Fine adjustment of the AV delay to achieve optimal ventricular filling can easily be performed looking at the monitored filling and arterial pressure values in the intensive care unit (ICU). Dyssynchrony carried by inappropriate single site ventricular pacing in the instance of AV block should further be avoided and available data suggest that temporary pacing from the systemic ventricular apex preserves systolic function ${ }^{16}$ and may be an important part of a prosynchronisation strategy. Resynchronisation pacing can usually be discontinued early in the postoperative course after spontaneous improvement in myocardial function.

The following case will demonstrate one facet of temporary CRT use in the postoperative period. A 4-month-old girl was admitted to the paediatric cardiac ICU after an otherwise uncomplicated primary repair of tetralogy of Fallot using a transannular patch. She was in overt right ventricular failure with a central venous pressure of $15 \mathrm{mmHg}$ and a mean arterial pressure between $40-50 \mathrm{mmHg}$, despite high dose catecholamine support and preload optimisation. The ECG revealed sinus rhythm with a slightly prolonged PR interval of $160 \mathrm{~ms}$ and complete right bundle branch block. A temporary pacing wire was placed on the right ventricular outflow tract during the operation and atrial synchronised ventricular pacing was started in an attempt to functionally alleviate the right bundle branch block causing RV desynchronisation and contributing potentially to RV failure. This led to an immediate increase in blood pressure (Figure 5), enabled reduction in inotropic support and volume replacement, and stabilised the haemodynamics during the first $48 \mathrm{~h}$ after surgery, after which pacing could be uneventfully discontinued.

\section{Permanent CRT}

In contrast to temporary resynchronization, permanent CRT has so far been reported only for the systemic ventricle. There are no data or indication criteria for permanent resynchronisation of the subpulmonary RV. Results relevant to paediatric and congenital heart disease patients can be retrieved from two larger retrospective surveys ${ }^{3,4}$ and a limited number of smaller studies. ${ }^{10}$ The following findings are notable:

> The majority of the patients reported in the two collaborative surveys ${ }^{3,4}$ were categorised as NYHA class II reflecting a more liberal and pro-active approach to CRT as compared to the current official indications. Such an approach seems to be supported by recent data from ischaemic and idiopathic cardiomyopathy showing successful reverse ventricular remodelling after CRT in an NYHA class II population. ${ }^{17}$

> The best response to CRT, with almost complete reverse remodelling, has been observed in patients with systemic left ventricles who were upgraded to CRT from conven-

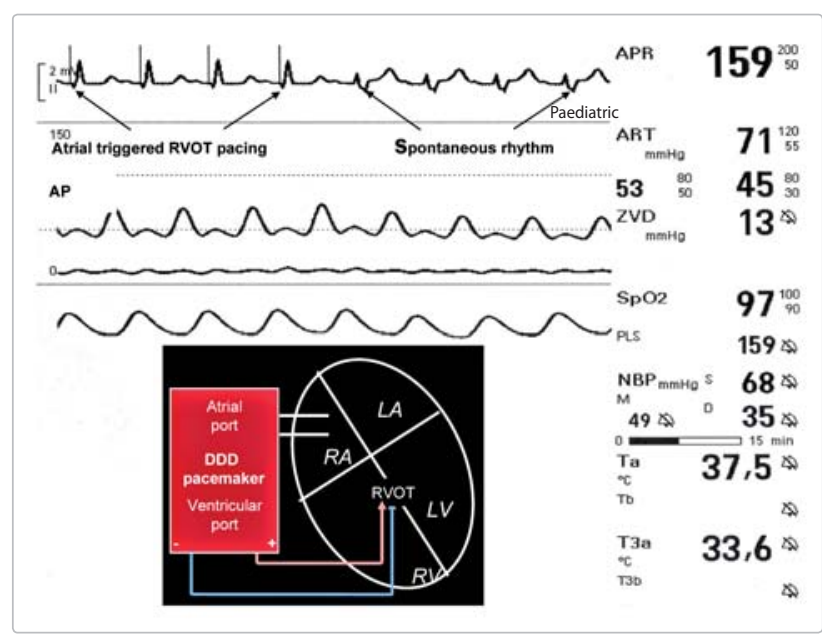

Figure 5 Haemodynamic effect of right ventricular resynchronisation for acute heart failure after repair of tetralogy of Fallot. Left side of the intensive care unit monitor printout displays atrial triggered right ventricular outflow tract pacing (see scheme at the bottom of the figure) leading to functional alleviation of the right bundle branch block (narrow QRS) and presumed improvement in right ventricular function. After pacing is switched off (right side of the panel) immediate haemodynamic deterioration is observed with a decrease of systolic arterial pressure by $10-15 \mathrm{mmHg}$.

AP - arterial pressure, LA - left atrium, LV - left ventricle, RA - right atrium, RV - right ventricle, RVOT - right ventricular outflow tract 
tional RV pacing. This cohort had significantly improved LV ejection fractions, increasing from a median of $21 \%$ to $53 \%(p<0.001)$ in studies with available individual data. ${ }^{10}$ This finding suggests the possibility of purely RV pacing induced LV failure completely reversible by CRT or, as recently reported, by the change of the pacing site towards the LV apex. ${ }^{18}$

> CRT in this group may help to delay heart transplantation. Almost $40 \%$ of the heart transplant candidates could be de-listed after $\mathrm{CRT}^{10}$ suggesting that all patients awaiting transplantation should be specifically screened for the presence of mechanical dyssynchrony as a potential substrate for improvement by CRT.

> Improvement in systolic ventricular function was independently predicted by the presence of a systemic LV and was better than for systemic RV. 4 This finding supports the influence of additional specific factors in the development of systemic RV failure, as reported elsewhere, such as different RV architecture, structural tricuspid valve regurgitation, and decreased myocardial perfusion reserve which cannot be corrected by CRT.

> The presence of primary dilated cardiomyopathy and a high NYHA class seems to predict non-response to CRT. ${ }^{4}$ This finding is in contrast to the adult idiopathic dilated cardiomyopathy population, where reported results are more favourable. The reason may lie in different aetiology of dilated cardiomyopathy in the young, including metabolic disease, muscular dystrophy and non-compaction, where the natural history is unfavourable. High NYHA class as a risk factor for CRT non-response probably argues in favour of offering resynchronisation therapy at an earlier stage of heart failure development to prevent irreversible ventricular deterioration.

The following two case reports reflect an example of permanent CRT utilisation in congenital heart disease patients.

\section{Case 1}

A 2.6-year-old girl who had undergone a patch closure of a ventricular septal defect in infancy resulting in complete heart block and requiring epicardial DDD pacemaker implantation was admitted because of progressive heart failure (NYHA III) despite full anticongestive therapy. The echocardiogram showed severely dilated left ventricle (LV end-diastolic volume index $=249 \mathrm{ml} / \mathrm{m}^{2}$ of body surface area) and an ejection fraction of $22 \%$ along with severe LV dyssynchrony reflected by a septal to posterior wall motion delay of 270 ms (see Figure 2 for explanation). The ECG revealed a paced QRS pattern with left bundle branch block morphology and $160 \mathrm{~ms}$ duration consistent with epicardial pacing from the RV free wall. An upgrade to biventricular pacing was performed by surgical placement of an epicardial LV free wall lead (Figure 6) and produced immediate abolition of the septal to posterior dyssynchrony. After 12 months of therapy complete reverse LV remodelling could be achieved with an LV end-diastolic volume index of $58 \mathrm{ml} / \mathrm{m}^{2}$ of body surface area and en ejection fraction of $60 \%$ (Figure 7). In this case, LV failure was solely caused by RV pacing induced dyssynchrony and CRT was the method of choice to treat this patient.

\section{Case 2}

A 12-year-old boy, who had undergone a Senning procedure for d-transposition of the great arteries, patch closure of ventricular septal defect and LV based DDD pacing for complete surgical heart block, experienced gradual deterioration of systemic RV function reflected by an echocardiographically measured fractional area of change of $12 \%$ along with grade 2 tricuspid regurgitation, being in NYHA class II. Because of a paced QRS duration of $190 \mathrm{~ms}$, the patient was upgraded to biventricular pacing by implanting an epicardial RV free wall lead at an area of late local electrical activation (170 ms after QRS onset, Figure 8), and pulmonary artery banding was performed concurrently aimed at retraining the RV for a late arterial switch operation. When biventricular pacing was switched on there was an immediate improvement in RV maximum $+\mathrm{dP} /$ dt from 496 to $821 \mathrm{mmHg} / \mathrm{s}$. Seventeen months after surgery the RV fractional area of change increased to $33 \%$, tricuspid regurgitation decreased by 0.5 grade, and significant RV reverse remodelling in terms of a decrease in the end-diastolic $\mathrm{RV}$ area from 34 to $25 \mathrm{~cm}^{2} / \mathrm{m}^{2}$ of body surface area could be
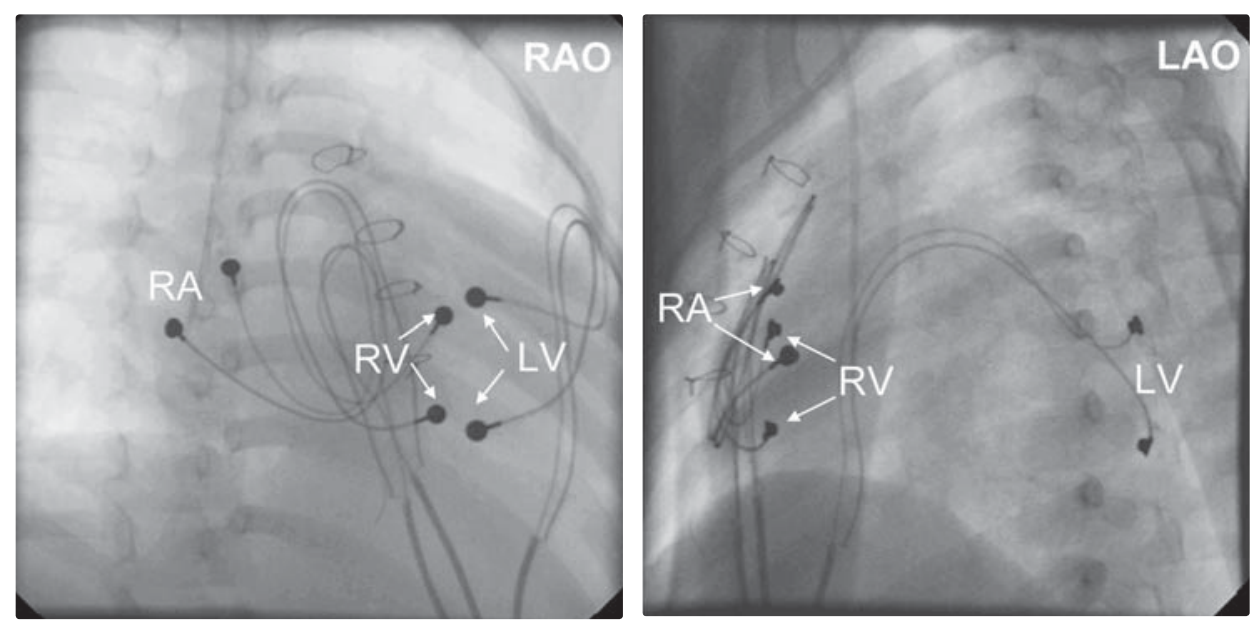

Figure 6 Upgrade to biventricular pacing for right ventricular (RV) free wall pacing induced left ventricular (LV) failure. The LV lead is placed at the border between the basal and mid ventricular posterior LV segment clearly spatially separated from RV lead. LV lies between the two ventricular leads and is activated by two fusing activation wave fronts. LAO - left anterior oblique projection, LV - left ventricle, $\mathrm{RA}$ - right atrium, $\mathrm{RAO}$ - right anterior oblique projection, $\mathrm{RV}$ - right ventricle 


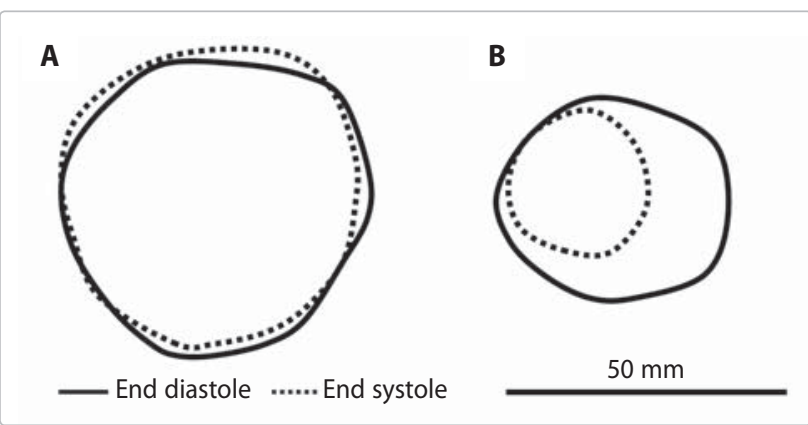

Figure 7 Endocardial end-diastolic and end-systolic contours of the left ventricle (LV) from the echocardiographic parasternal short axis image. (A) Before cardiac resynchronisation therapy. Poor LV contractility is seen along with a twisting movement with absence of concentric contraction. (B) Complete reverse remodelling after 12 months of therapy.

observed. Given the concomitant functional improvement to NYHA class I the plan for late arterial switch was deferred. This case probably best illustrates the complexity of decision making in systemic RV patients. Combined strategies to improve RV function by resynchronisation and to decrease tricuspid regurgitation by pulmonary artery banding or valve reconstruction/replacement may hold promise for certain selected patients.

\section{Conclusion}

CRT is a valuable tool for treating dyssynchrony associated acute and chronic heart failure in patients with paediatric or congenital heart disease. The anatomic and functional heterogeneity of this population calls for individual approaches in both the indication process

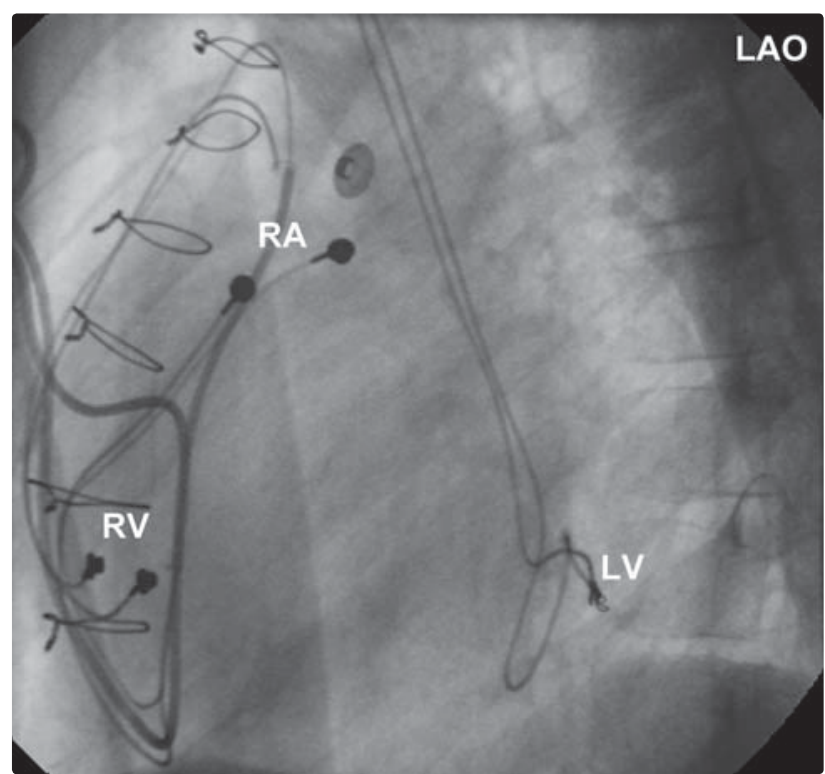

Figure 8 Example of mixed lead placement used for biventricular pacing in a patient after a Senning operation for transposition of great arteries. A transvenous left ventricular lead is used along with an epicardial right ventricular free wall lead and epicardial right atrial lead. Systemic right ventricle lies between the two ventricular leads and is activated by two fusing activation wave fronts.

LAO - left anterior oblique projection, LV - left ventricle, RA - right atrium, RV - right ventricle

and CRT implementation. Achievable results depend on the underlying substrate but may reach up to successful discontinuation from cardiopulmonary bypass in the acute postoperative heart failure setting, complete reverse remodelling of the systemic ventricle in permanent CRT, or delisting from the heart transplant waiting list. Ventricular

\section{CRT in congenital heart disease: key points}

\section{Pathophysiology of electromechanical dyssynchrony}

Sequence of events caused by electromechanical dyssynchrony:

- Dyssynchronous ventricular contraction with early and late contracting segments

- Late contracting segments are stretched by the early contracting regions and perform a higher local myocardial work.

- This work is to some part wasted because late contraction appears after semilunar valve closure and end of the ventricular ejection phase.

- Inefficient ventricular contraction and pathologic remodelling results.

\section{Electromechanical dyssynchrony}

Causes of clinically relevant electromechanical dyssychrony differ from adult idiopathic and ischaemic cardiomyopathy. The following are most prevalent:

- Dyssynchrony associated with cardiac pacing from the subpulmonary ventricle.

- Dyssynchrony caused by right bundle branch block.

The combination of systemic left ventricle along with a left bundle branch block is rare in the congenital heart disease population.

\section{Principles of CRT}

CRT is based on electrical pre-excitation of late contracting myocardial segments and causes:

- Correction of septal to lateral intraventricular dyssynchrony.

- Immediate increase in contraction efficiency by abolition of wasted myocardial work.
- Mechanical AV delay optimisation and improvement of ventricular filling

- Reverse ventricular remodelling.

- Functional improvement.

\section{Temporary CRT}

Prerequisites of successful use of temporary CRT for improvement of cardiac output in patients with acute heart failure after surgery for congenital heart defects are:

- Identification of the failing dyssynchronous ventricle.

- Placement of temporary ventricular pacing wires close to segments with late contraction (cooperation with the surgeon).

- Appropriate external pulse generator programming and haemodynamic optimisation of the AV delay.

- Use of temporary CRT in the operating room if there are problems with disconnection from cardiopulmonary bypass (cooperation between surgeon, anaesthetist, intensivist and electrophysiologist).

\section{Permanent CRT}

Permanent CRT is effective in the treatment of systemic ventricular dysfunction and failure associated with intraventricular mechanical dyssynchrony:

- Improvement after CRT is better in systemic LV patients and in conventional pacing induced dyssynchrony.

- Combined strategies aimed at resynchronisation and surgical improvement or relief of tricuspid regurgitation may be helpful in systemic RV patients.

- Heart transplant candidates should specifically be screened for mechanical dyssynchrony as a CRT correctable cause of heart failure. 


\section{You can get CPD/CME credits for Education in Heart}

Education in Heart articles are accredited by both the UK Royal College of Physicians (London) and the European Board for Accreditation in Cardiology - you need to answer the accompanying multiple choice questions (MCQs). To access the questions, click on BMJ Learning: Take this module on BMJ Learning from the content box at the top right and bottom left of the online article. For more information please go to: http://heart.bmj.com/misc/education.dtl

- RCP credits: Log your activity in your CPD diary online (http:// www.rcplondon.ac.uk/members/CPDdiary/index.asp) - pass mark is $80 \%$.

- EBAC credits: Print out and retain the BMJ Learning certificate once you have completed the MCQs - pass mark is 60\%. EBAC/EACCME Credits can now be converted to AMA PRA Category 1 CME Credits and are recognised by all National Accreditation Authorities in Europe (http://www.ebac-cme.org/newsite/?hit=men02).

Please note: The MCQs are hosted on BMJ Learning - the best available learning website for medical professionals from the BMJ Group. If prompted, subscribers must sign into Heart with their journal's username and password. All users must also complete a one-time registration on BMJ Learning and subsequently log in (with a BMJ Learning username and password) on every visit.

dyssynchrony is generally a correctable cause of ventricular dysfunction and should not be forgotten in any patient with acute or chronic heart failure. Available paediatric review articles may be of further help to the interested reader. ${ }^{10,19,20}$

Competing interests: In compliance with EBAC/EACCME guidelines, all authors participating in Education in Heart have disclosed potential conflicts of interest that might cause a bias in the article. The author has no competing interests.

\section{References}

1. Swedberg K, Cleland J, Dargie H, et al; Task Force for the Diagnosis and Treatment of Chronic Heart Failure of the European Society of Cardiology. Guidelines for the diagnosis and treatment of chronic heart failure: executive summary (update 2005). Eur Heart J 2005;26:1115-1140.

- Current European recommendations for the use of CRT in chronic LV failure.

2. American College of Cardiology/American Heart Association. A Report of the American College of Cardiology/American Heart Association Task Force on Practice Guidelines. ACC/AHA 2005 guideline update for the diagnosis and management of chronic heart failure in the adult - summary article. J Am Coll Cardiol 2005;46:1116-1143.

3. Dubin AM, Janoušek J, Rhee $E$, et al. Resynchronization therapy in pediatric and congenital heart disease patients: an international multicenter study. J Am Coll Cardiol 2005;46:2277-2283.

- First larger survey on the use of CRT in paediatric and congenital heart disease.

4. Janoušek J, Grollmuss O, Abdul-Khaliq $\mathrm{H}$, et al. Predictors of response to cardiac resynchronization therapy (CRT) in pediatric and congenital heart disease: subanalysis of a retrospective European multicenter study. Cardiol Young 2007;17(Suppl. 1):16.[abstract]
5. Wyman BT, Hunter WC, Prinzen FW, et al. Mapping propagation of mechanical activation in the paced heart with MRI tagging. Am J Physiol 1999;276:H881-H891

- Important pathophysiologic insight into mechanical cardiac dyssynchrony.

6. Prinzen FW, Hunter WC, Wyman BT, et al. Mapping of regional myocardial strain and work during ventricular pacing: experimental study using magnetic resonance imaging tagging. J Am Coll Cardiol 1999;33: 1735-1742.

7. van Oosterhout MF, Prinzen FW, Arts T, et al. Asynchronous electrical activation induces asymmetrical hypertrophy of the left ventricular wall. Circulation 1998;98:588-595.

8. Janoušek J, Tomek V, Chaloupecký V, et al. Dilated cardiomyopathy associated with dual-chamber pacing in infants: improvement through either left ventricular cardiac resynchronization or programming the pacemaker off allowing intrinsic normal conduction. J Cardiovasc Electrophysiol 2004;15:470-474.

9. Nelson GS, Berger RD, Fetics BJ, et al. Left ventricular or biventricular pacing improves cardiac function at diminished energy cost in patients with dilated cardiomyopathy and left bundle-branch block. Circulation 2000;102:3053-3059.

- First study confirming energetic efficiency of CRT.

10. Janoušek J, Gebauer RA. Cardiac resynchronization therapy in pediatric and congenital heart disease. Pacing Clin Electrophysiol 2008;31(Suppl 1): S21-S23.

- Short review on currently available studies on temporary and permanent CRT in the young.

11. Diller GP, Okonko D, Uebing A, et al. Cardiac resynchronization therapy for adult congenital heart disease patients with a systemic right ventricle: analysis of feasibility and review of early experience. Europace 2006;8:267-272.

12. Kass DA. An epidemic of dyssynchrony: but what does it mean? J Am Coll Cardiol 2008;51:12-17.

- Thorough review of different aspects of mechanical dyssynchrony with respect to CRT.

13. Rao RK, Kumar UN, Schafer J, et al. Reduced ventricular volumes and improved systolic function with cardiac resynchronization therapy: a randomized trial comparing simultaneous biventricular pacing, sequential biventricular pacing and left ventricular pacing. Circulation 2007;115:2136-2144.

14. Chung ES, Leon AR, Tavazzi L, Sun JP, et al. Results of the Predictors of Response to CRT (PROSPECT) trial. Circulation 2008;117:2608-2616.

15. Gorcsan J $3^{\text {rd }}$, Tanabe M, Bleeker GB, et al. Combined longitudinal and radial dyssynchrony predicts ventricular response after resynchronization therapy. J Am Coll Cardiol 2007;50:1476-1483.

- Recent study confirming utility of echocardiographic speckle tracking in prediction of CRT response.

16. Vanagt WY, Verbeek XA, Delhaas T, et al. Acute hemodynamic benefit of left ventricular apex pacing in children. Ann Thorac Surg 2005;79:932-936.

- First human study showing acute haemodynamic benefit of left ventricular apical pacing.

17. Landolina M, Lunati M, Gasparini M, et al; InSync/InSync ICD Italian Registry Investigators. Comparison of the effects of cardiac resynchronization therapy in patients with class II versus class III and IV heart failure (from the InSync/InSync ICD Italian Registry). Am J Cardiol 2007;100: 1007-1012.

18. Tomaske M, Breithardt OA, Balmer $C$, et al. Successful cardiac resynchronization with single-site left ventricular pacing in children. Int J Cardiol 2009;136:136-143.

19. Dubin AM. Resynchronization in pediatrics: who needs it? J Am Coll Cardiol 2005:46:2290-2291.

20. Khairy P, Fournier A, Thibault B, et al. Cardiac resynchronization therapy in congenital heart disease. Int J Cardiol 2006;109:160-168. 